\title{
The future of neuro-oncology
}

\author{
Joseph M. Piepmeier
}

Received: 13 July 2009 /Accepted: 15 July 2009 /Published online: 29 July 2009

(C) Springer-Verlag 2009

\begin{abstract}
Summary
Over the past 25 years, there has been some modest progress in improving survival (in months) of patients with malignant gliomas. Much of this improvement is the result of better imaging techniques that permit safer, more aggressive surgical options. However, limitations imposed by tumor location and infiltration remain a challenge that cannot be addressed by surgeons. The current standard of care (Stupp Protocol) has been shown to produce limited survival advantages, but significant changes in outcome await better methods of drug delivery, patient selection for specific treatment, and more accurate methods of determining how well a patient is responding to therapy. Recent findings have introduced possible methods of addressing these continued challenges. This review will examine some of the more recent data and address where new therapies may have a more dramatic effect in controlling this disease.
\end{abstract}

\section{Surgery}

The first multi-institutional randomized clinical trial for malignant gliomas (Brain Tumor Study Group [BTSG] 6901) demonstrated that surgery alone had minimal effect on survival and the addition of an alkylating agent (bis-chloronitrosourea [BCNU]) did not significantly improve this poor outcome ( $<6$ months) [20]. The

Presented at the Academia Eurasiana Neurochirurgica, 15th Convention, Istanbul, Turkey 2009.

J. M. Piepmeier $(\bowtie)$

Department of Neurosurgery, Yale School of Medicine,

Yale University,

333 Cedar Street,

New Haven, CT 06520, USA

e-mail: joseph.piepmeier@yale.edu addition of radiation therapy increased survival to around 9 months. The more recent data from the subsequent clinical trial directed by the European Organisation for Research and Treatment of Cancer (EORTC) produced a median survival of 14.6 months when radiation therapy was utilized along with a different alkylating agent, Temodar [16]. A comparison of the survival curves from these large clinical trials shows that there is a significant improvement in the early mortality following surgery in the latter study (Fig. 1). The 65\% early survival (6 months) noted in the BTSG reached $85 \%$ in the EORTC study. There are several reasons why improvement in outcome has been achieved over the past 25 years. Radiation delivery and dosimetry performed in 1978 are similar to techniques utilized in 2005. Temodar is a better agent than $\mathrm{BCNU}$ in the treatment of malignant gliomas, however, they are both alkylating agents. One conclusion is that the improvement in outcome from 1978 to 2005 , in part, is a result of safer and more complete surgical cytoreduction producing minimal tumor burden for adjuvant treatment.

Improved surgical mortality and morbidity has been a direct result of more informative imaging. The common utilization of multiple sequences from magnetic resonance imaging (MRI), diffusion tensor imaging (DTI), MR spectroscopy, positron emission tomography (PET), and functional imaging has increased the surgeons' confidence in identifying tumor and protecting the brain (Fig. 2). Intraoperative cortical and subcortical mapping along with intraoperative imaging with MRI or computed tomography (CT) have enabled surgeons to increase the extent of resection while preserving function, but surgery has a limited capacity for tumor control. A more recent study comparing the progression-free survival in patients that received fluorescence-guided identification of tumor (5ALA) to those receiving conventional surgery also suggest that more aggressive resection can modestly increase 


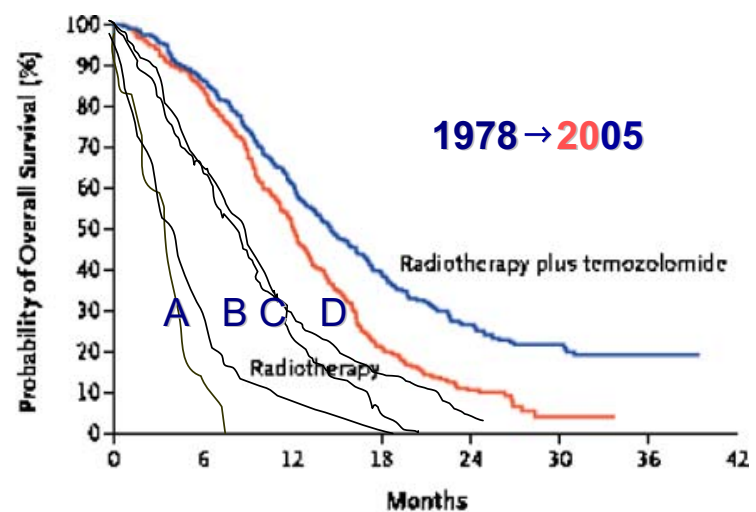

$\begin{array}{llllrrrr}\begin{array}{l}\text { No, at Risk } \\ \text { Radiotherapy }\end{array} & 286 & 240 & 144 & 59 & 23 & 2 & 0 \\ \begin{array}{c}\text { Radiotherapy } \\ \text { plus tems. } \\ \text { zolomide }\end{array} & 287 & 246 & 174 & 109 & 57 & 27 & 6\end{array}$

Fig. 1 Survival curves comparing outcome from BTSG 6901 [20] and EORTC [16]. Courtesy of H. Richard Winn, MD

survival [15]. What is also impressive from this study is that, while all patients were considered to have lesions that could be removed, gross total resection was achieved in only $36 \%$ of patients using conventional techniques and in $65 \%$ utilizing fluorescence guidance. Further improvements in surgical navigation and tumor resection will continue to improve outcomes and extent of resection. However, the limitations of surgical therapy for an infiltrative disease in the brain strongly indicate that dramatic improvement in tumor control requires innovative strategies for radiationand chemotherapy-resistant glioma cells.

\section{Imaging}

One of the major goals in future treatment is determining tumor responsiveness to treatment. It is clear that anatomic
MRI is insufficiently specific to meet this need. The current standard of quantifying responsiveness (McDonald Criteria) relies on volumetric measurements of regions of contrast enhancement [18]. It has been recognized for many years that postsurgical and radiation enhancement can result from inflammatory cells thus reducing the specificity of MRI to accurately measure the extent of the lesion. The concept of pseudoprogression (increased enhancing tissue following radiotherapy) has further confounded our capacity to determine how a tumor has responded to treatment. This is particularly true of tumors that contain methylation of the MGMT promoter [1]. In addition, the current use of antiVEGF agents has been shown to dramatically reduce enhancement while the cytotoxic effect on tumor cells remains less certain. Future imaging techniques may utilize metabolic differences between the tumor and the brain to address this need. Initial studies have shown that metabolically active tumors can become inactive while the anatomic imaging (MRI) shows continued stability. These data suggest that imaging parameters that rely on revealing an abnormal blood-brain barrier or increased water content in the tissue simply cannot reliably detect the effects of treatment on the tumor. ${ }^{11} \mathrm{C}$-methionine PET may provide sufficient discrimination between active tumor and brain to help determine responsiveness, but this parameter is also dependent on the relative amount of microvascular density $[8,12]$. Longitudinal studies in patients with high-grade oligodendrogliomas may reveal different parameters than patients with high-grade astrocytomas. These diagnostic classifications require further clarification of baseline metabolic parameters for future use of PET.

Initial investigations of MR spectroscopy have focused on choline, creatine, and $\mathrm{N}$-acetylaspartate as parameters to measure tumor responsiveness. These results have been insufficient to discriminate tumor from treatment-related changes because of the relative variations in these

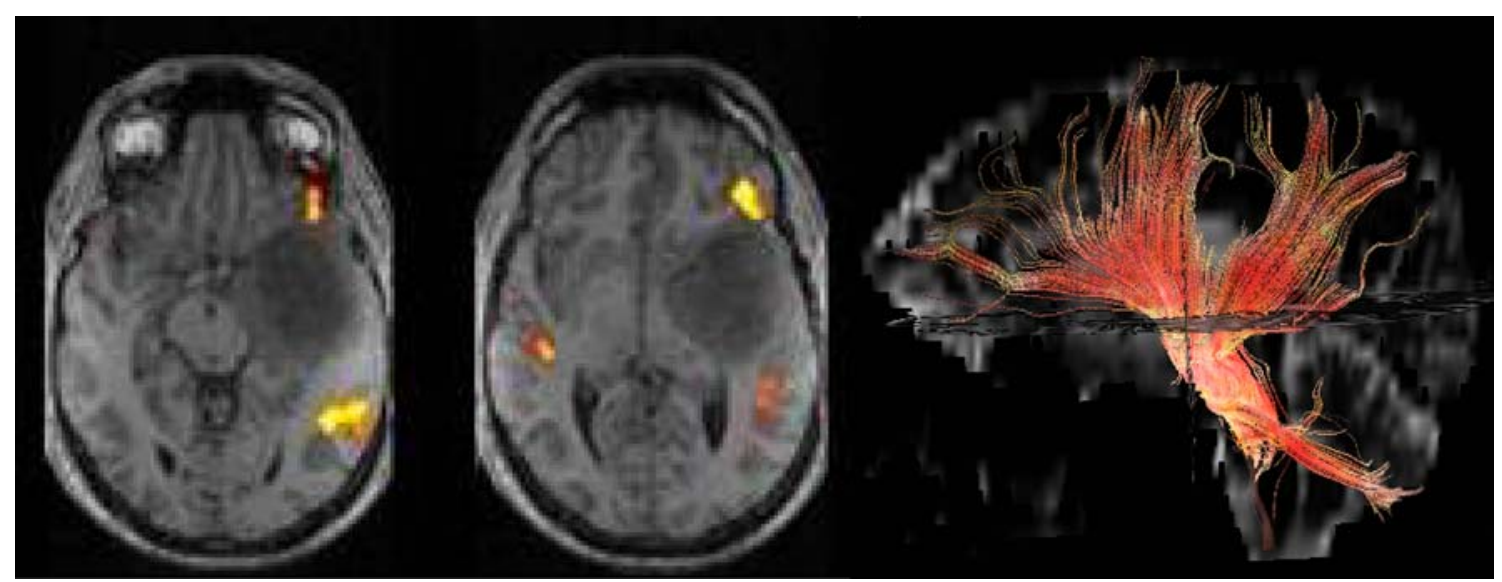

Fig. 2 Left functional MRI for language revealing primary cortical regions (Broca's and Wernicke's areas) for sensory and motor speech. Right MRI tractography demonstrating motor and sensory fibers displaced by a tumor in the subcortical white matter 
metabolites attributed to inflammatory cells also overlap with tumor cells [3]. Recently, we have found that glutamine is a more specific spectral metabolite for tumor cells. Standard MRI magnet strengths (1.5 and $3 \mathrm{~T})$ do not provide sufficient resolution for measuring glutamine. Our work with a 7-T magnet indicates that not only can glutamine be accurately measured, but also that this parameter is significantly increased in tumor tissue and not in inflammatory tissue (Fig. 3). We are continuing to investigate glutamine as a more reliable and specific marker for tumor responsiveness.

Ultimately, it is apparent that anatomic MRI will need to be replaced as a method for measuring the effectiveness of glioma therapy and that the McDonald Criteria will be replaced by a more sensitive and specific imaging technique. Metabolic imaging may provide an answer to this problem.

\section{Tumor classification: reductionism}

The identification of codeletion of $1 \mathrm{p} / 19 \mathrm{q}$ as a genetic marker that identifies a subgroup of high-grade oligodendrogliomas with less aggressive growth and better responsiveness to therapy has stimulated a search for additional molecular and genetic characteristics that help to define patient selection for specific treatment $[2,7]$. These studies have provided valuable information on the spatial and temporal genetic variations that characterize malignant gliomas. More recently, it has been shown that the association between $1 \mathrm{p}$ and $19 \mathrm{q}$ is often the result of a translocation [5]. The identification of the association between $1 \mathrm{p}$ deletion and stathmin (a microtubule-associated protein located on $1 \mathrm{p}$ ) suggest that the biological significance of this genetic anomaly resides in the first chromosome and that the associated $19 \mathrm{q}$ loss is of little consequence.

Tumor

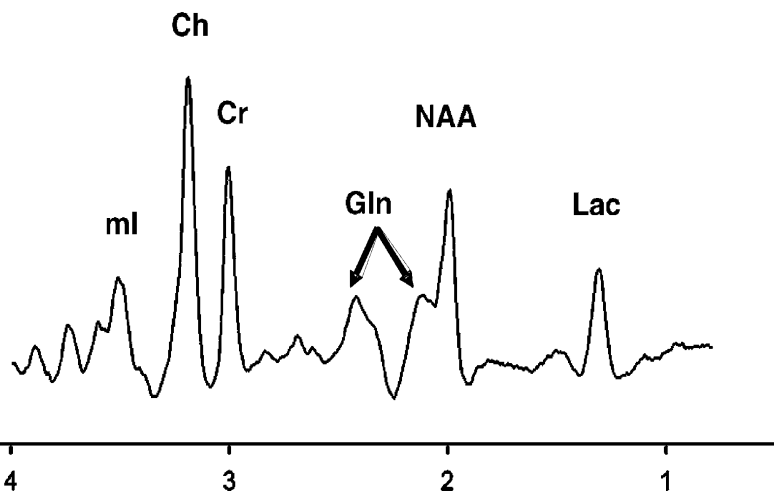

Fig. 3 MR spectrograph of a malignant glioma obtained from a 7-T magnet. Note the peaks from glutamine $(G l n)$
More recently, laboratories in North America and Europe have identified mutation of the isocitrate dehydrogenase 1 gene (IDH1) as an early event in the evolution of low-grade gliomas $[4,10,17,22]$. A single amino acid mutation on IDH1 is present in $70-90 \%$ of astrocytomas and oligodendrogliomas and is also found in malignant gliomas that evolve from a lower-grade lesion. Initial survival comparisons indicate that IDH1 mutation confers a better prognosis for survival. This difference may be a result of age differences between patients with IDH1 mutation and those without it. However, these studies may produce a useful marker for anticipating outcome and prognosis. Further work is needed to explain the biological effects of IDH1 on the biology of gliomas.

In 2008, the Cancer Genome Atlas Research Network published a comprehensive examination of a multidimensional analysis of molecular characteristics in 206 glioblastomas [2]. An integrated assessment of common genetic mutations revealed frequent alterations in three major pathways, RTK/RAS/PI3K, p53, and RB. Within each of these major groups, numerous amplifications, mutations, and deletions were found. The complexity of genomic analysis is further indicated by the observation that a majority (74\%) of glioblastomas harbor alterations in all major pathways. Consequently, any therapy that is designed to address a single pathway mutation is destined to increase selection pressure for the emergence of cells containing alternative dysregulation. The ability to address all of these problems confounds monotherapy strategies and raises the necessity for multiple agent treatment regimens.

In 2009, the publication of an unsupervised analysis of transcriptomic profiles in 159 gliomas revealed further subclassification of these tumors into two major groups (oligodendroglioma-rich and glioblastoma-rich) with further subclassification into six prognostic groups [7]. These studies utilize large tissue banks from a variety of sources to try to further characterize malignant gliomas by their molecular and genetic profiles. A critical component of these studies is the multiplicity and variability in genetic alterations within each tumor. Genetic chaos seems to be a fundamental confounding challenge for treatment based on reversing pathway regulatory mechanisms.

Scientific investigation commonly approaches largescale problems by reducing them into a series of smaller questions that can be managed. The studies noted above demonstrate a reductionist approach to defining malignant gliomas. This has led to some proof of principle (combined epidermal growth factor receptor [EGFR]/ mTOR kinase inhibition has a beneficial effect on tumor growth in PTEN deficient gliomas), but has yet to significantly alter the long-term prognosis for glioblastoma patients [21]. A reductionist approach has significant limitations based on the need to address multiple pathways 
of growth dysregulation that require multiagent therapy to counteract these effects.

\section{Novel therapy}

An alternative approach is to devise a treatment that is not dependent on or subject to the changing and variable genome, but to attack the tumor based on general characteristics that are present in the majority of tumors. This strategy has focused several approaches ranging from developing a tumor vaccine to convection-enhanced delivery of tumor-specific toxins as well as selecting glioma stem cells as the target of therapy. In addition, the ability to effectively deliver agents into the tumor as well as the brain remains a challenge.

\section{Tumor-specific targets}

Current clinical trials include the use of TransMID 107 (transferrin-CRM107). This is an immunotoxin that is a conjugate of CRM107 and transferrin. It is known that transferrin receptors are expressed on all rapidly dividing cells, including tumors, with minimal expression in nondividing tissues such as normal brain. Another study is investigating Cotara $^{\circledR}$, a monoclonal antibody conjugated with ${ }^{131}$ I designed to bind to DNA that is exposed only on dead and dying cells [16]. It is delivered using convectionenhanced delivery. The PRECISE trial, Phase III Randomized Evaluation of CED of IL13-PE38QQR (CB) Compared to Gliadel Wafers with Survival Endpoint in Glioblastoma (GBM) Patients at First Recurrence failed to demonstrate a survival benefit for the investigational agent [6]. This may be the result of problems in distribution of the agent into the areas of tumor infiltration. Improvements in convection-enhanced delivery will be required before this strategy can achieve clinical use.

We have established a collaborative effort with biomedical engineering to design and test biodegradable nanoparticles as a vehicle to deliver tumor-specific toxins to infiltrative tumors (Fig. 4). The goal of this project is to enhance safety by developing agents that attack the tumor and not attach to the brain. CED is also seen as a potentially effective method for delivering these particles into the brain around a surgical resection area.

\section{Immunotherapy}

A glioma vaccine may ultimately provide effective treatment for malignant gliomas. CDX-110 ${ }^{\circledR}$ is an immunotherapy that targets the tumor-specific molecule called EGFRvIII, a

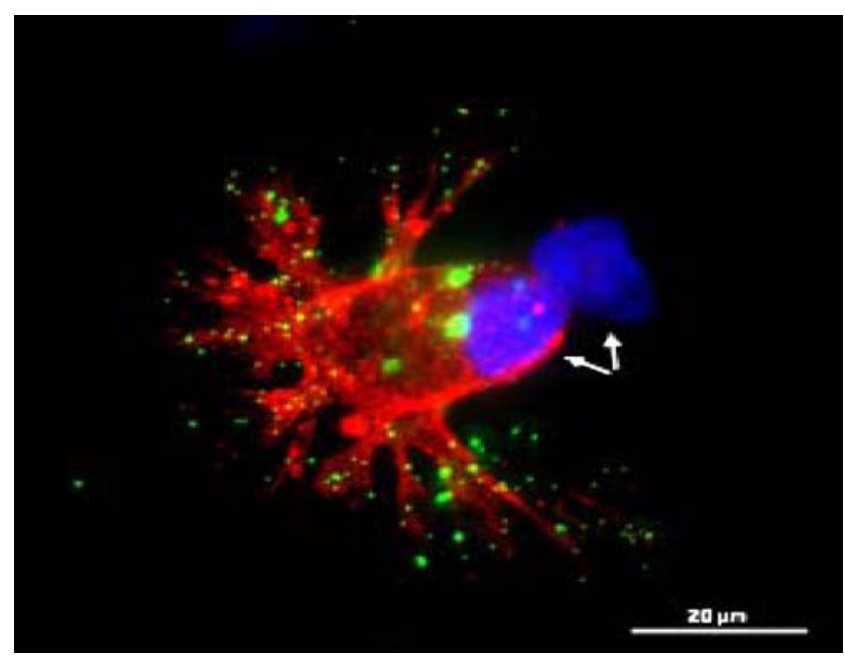

Fig. 4 Photograph of two nuclei (arrows) from a glioma cell (red) and a normal astrocyte (unlabeled). Nanoparticles (green) carrying a tumor-specific antibody bind with the tumor cell and not the astrocyte

functional variant of the EGFR, a protein that has been well validated as a target for cancer therapy. DCVax ${ }^{\circledR}$-Brain utilizes a patient's own dendritic cells and an extract of the patient's own tumor cells to achieve an immune response. Oncophage ${ }^{\circledR}$ is a vaccine made from individual patients' glioma targeting heat shock proteins. Each of these clinical studies has substantial preclinical data to show potential for a desired immune response to the tumor. Each also has selected a different target antigen with relative variations in specificity for glioma or a more general target found in malignant tissue. The immunosuppressive effects of a glioma pose some limitations on this strategy. Tumor control will require a sufficient immune stimulation and migration of effector immune cells into the lesion. These challenges continue to pose significant barriers to current studies.

One approach is to develop a biologically active agent that is selective for tumor tissue and can reach invasive cells. We have investigated the use of an oncolytic replication-competent virus for glioma treatment [9]. This work has produced a glioma-adapted vesicular stomatitis virus strain (VSVrp30) that is effective in vitro and in an animal model (Fig. 5). Intravenous VSV infects intracranial tumor implants at multiple sites as well as distant migrating tumor cells. Further potential clinical use of this agent requires additional toxicity studies in primates to confirm safety for human trials.

\section{Cancer stem cells}

There are intriguing findings that indicate that gliomas may contain a population of progenitor cells or cancer 


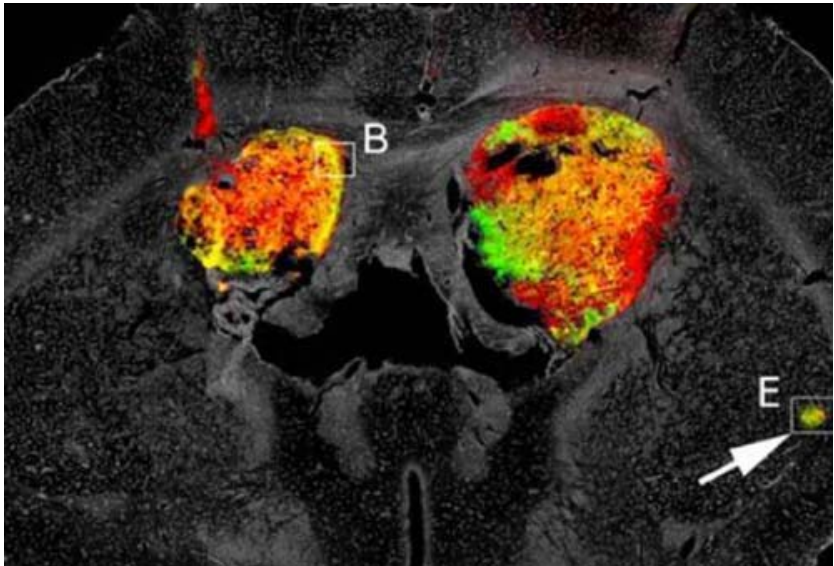

Fig. 5 Photograph of mouse brain implanted with human glioma cells (red) following systemic injection of VSVrp30 (green). Note the oncolytic virus has attached to and proliferated in the implants $(B)$. A small tumor satellite $(E)$ was also attacked by the virus

stem cells that are more resistant to radiotherapy and chemotherapy than the more lineage-defined cells $[11,13$, 14, 19, 23]. These stem cells may be a source of gliomas and may be one of the reasons these tumors fail to respond more effectively to current treatment. Since the initial publication suggesting that CD133 may be a marker for identifying glioma stem cells, several subsequent publications indicate that other markers also identify this population. In addition, there appears to be some significant differences in stem cells found in tumors compared with resident cells in the periventricular region as well as variations in proliferative capacity of glioma stem cells depending on which region of the tumor is sampled. Regardless, this subpopulation may be an important reason for current treatment failure.

We have directed a laboratory effort to isolate glioma stem cells from glioblastomas and to perform highthroughput chemosensitivity screening of numerous agents to detect agents that are cytotoxic to stem cells (Fig. 6). Our initial screening has focused on FDAapproved agents that were not necessarily designed for brain tumor therapy. This strategy is directed toward identifying effective drugs that have been safely used in prior human studies with established toxicity profiles. It is anticipated that this approach may provide a more costeffective therapy that will be accessible to a large population of tumor patients.

\section{Conclusion}

The current standard of care for a patient with a malignant glioma has produced modest increase in survival for these patients. Some of this improvement is the result of better surgical care reducing the early postoperative mortality and morbidity and providing the minimal tumor burden for adjuvant therapy. The search for a better understanding of the biology and genomics of gliomas has shown that a few identifiable biological markers can serve to predict which patients are more likely to have a better survival and to respond to treatment. Unfortunately, the temporal and spatial variability of genetic mutations that characterize the evolution of a malignant glioma and the multiplicity of alternative pathways that release tumor cells from normal regulatory mechanisms limit our ability to find effective treatment. This genomic instability, present in the majority of tumors, limits the long-term effectiveness of any treatment that focuses on epigenetic mutations as a target for tumor control. While initial tumor control can be demonstrated, treatment strategies focused on a specific pathway will produce selective advantages for resistance in regions of the tumor that contain alternative mutations.

An alternative approach is to generate treatment that is not susceptible to genomic instability and that is cytotoxic for all tumor cells. This strategy is appealing, but the lack of specificity can result in unacceptable toxicity. The optimal treatment must address these liabilities. In addition, drug delivery remains a challenge. Innovative treatment will only become successful if it can reach the intended target.

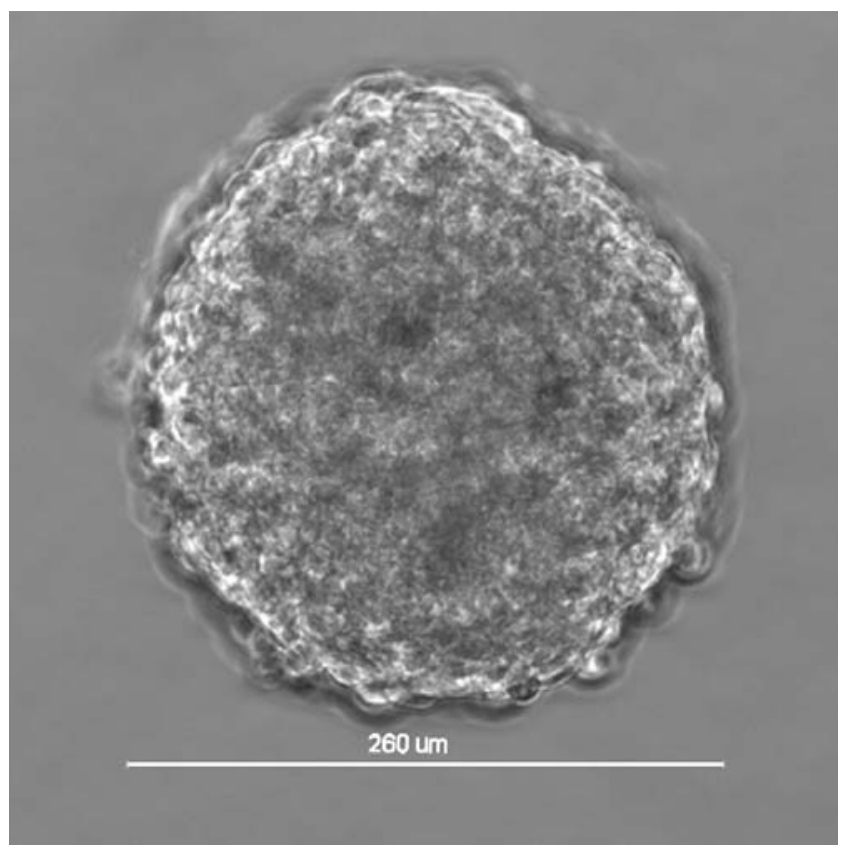

Fig. 6 Microsphere of glioma stem cells derived from human glioblastoma 


\section{References}

1. Brandes AA, Franceschi E, Tosoni A, Blatt V, Pession A, Tallini G, Bertorelle R, Bartolini S, Calbucci F, Andreoli A, Frezza G, Leonardi M, Spagnolli F, Ermani M (2008) MGMT promoter methylation status can predict the incidence and outcome of pseudoprogression after concomitant radiochemotherapy in newly diagnosed glioblastoma patients. J Clin Oncol 26(13):2192-2197

2. Cancer Genome Atlas Research Network (2008) Comprehensive genomic characterization defines human glioblastoma genes and core pathways. Nature 455(7216):1061-1068

3. DeBerardinis RJ, Mancuso A, Daikhin E, Nissim I, Yudkoff M, Wehrli S, Thompson CB (2007) Beyond aerobic glycolysis: transformed cells can engage in glutamine metabolism that exceeds the requirement for protein and nucleotide synthesis. Proc Natl Acad Sci U S A 104(49):19345-19350

4. Hartmann C, Meyer J, Balss J, Capper D, Mueller W, Christians A, Felsberg J, Wolter M, Mawrin C, Wick W, Weller M, HeroldMende C, Unterberg A, Jeuken JW, Wesseling P, Reifenberger G, von Deimling A (2009) Type and frequency of IDH1 and IDH2 mutations are related to astrocytic and oligodendroglial differentiation and age: a study of 1,010 diffuse gliomas. Acta Neuropathol (in press)

5. Jenkins RB, Blair H, Ballman KV, Giannini C, Arusell RM, Law M, Flynn H, Passe S, Felten S, Brown PD, Shaw EG, Buckner JC (2006) A $t(1 ; 19)(q 10 ; p 10)$ mediates the combined deletions of $1 p$ and $19 q$ and predicts a better prognosis of patients with oligodendroglioma. Cancer Res 66(20):9852-9861

6. Kunwar S, Prados M, Chang S, Berger M, Lang F, Piepmeier J, Sampson J, Ram Z, Gutin P, Gibbons R, Aldape K, Croteau D, Sherman J, Puri R (2007) Direct intracerebral delivery of Cintredekin Besudotox (IL13-PE38QQR) in patients with recurrent malignant glioma. J Clin Oncol 25(7):837-844

7. Li A, Walling J, Ahn S, Kotliarov Y, Su Q, Quezado M, Oberholtzer JC, Park J, Zenklusen JC, Fine HA (2009) Unsupervised analysis of transcriptomic profiles reveals six glioma subtypes. Cancer Res 69(5):2091-2099

8. Nojiri T, Nariai T, Aoyagi M, Senda M, Ishii K, Ishiwata K, Ohno K (2009) Contributions of biological tumor parameters to the incorporation rate of L: -[methyl-(11)C] methionine into astrocytomas and oligodendrogliomas. J Neurooncol 93(2):233-241

9. Ozduman K, Wollmann G, Piepmeier JM, van den Pol AN (2008) Systemic vesicular stomatitis virus selectively destroys multifocal glioma and metastatic carcinoma in brain. J Neurosci 28(8): 1882-1893

10. Patel SJ, Shapiro WR, Laske DW, Jensen RL, Asher AL, Wessels BW, Carpenter SP, Shan JS (2005) Safety and feasibility of convectionenhanced delivery of Cotara for the treatment of malignant glioma: initial experience in 51 patients. Neurosurgery 56(6):1243-5216

11. Piccirillo SG, Combi R, Cajola L, Patrizi A, Redaelli S, Bentivegna A, Baronchelli S, Maira G, Pollo B, Mangiola A, DiMeco F, Dalprà L, Vescovi AL (2009) Distinct pools of cancer stem-like cells coexist within human glioblastomas and display different tumorigenicity and independent genomic evolution. Oncogene 28(15):1807-1811
12. Pirotte BJ, Levivier M, Goldman S, Massager N, Wikler D, Dewitte O, Bruneau M, Rorive S, David P, Brotchi J (2009) Positron emission tomography-guided volumetric resection of supratentorial high-grade gliomas: a survival analysis in 66 consecutive patients. Neurosurgery 64(3):471-481

13. Sanai N, Alvarez-Buylla A, Berger MS (2005) Neural stem cells and the origin of gliomas. N Engl J Med 353(8):811-822

14. Singh SK, Clarke ID, Terasaki M, Bonn VE, Hawkins C, Squire J, Dirks PB (2003) Identification of a cancer stem cell in human brain tumors. Cancer Res 63(18):5821-5828

15. Stummer W, Pichlmeier U, Meinel T, Wiestler OD, Zanella F, Reulen HJ, ALA-Glioma Study Group (2006) Fluorescenceguided surgery with 5 -aminolevulinic acid for resection of malignant glioma: a randomised controlled multicentre phase III trial. Lancet Oncol 7(5):392-401

16. Stupp R, Mason WP, van den Bent MJ, Weller M, Fisher B, Taphoorn MJ, Belanger K, Brandes AA, Marosi C, Bogdahn U, Curschmann J, Janzer RC, Ludwin SK, Gorlia T, Allgeier A, Lacombe D, Cairncross JG, Eisenhauer E, Mirimanoff RO, European Organisation for Research and Treatment of Cancer Brain Tumor and Radiotherapy Groups, National Cancer Institute of Canada Clinical Trials Group (2005) Radiotherapy plus concomitant and adjuvant temozolomide for glioblastoma. N Engl J Med 352(10):987-996

17. Tchoghandjian A, Baeza N, Colin C, Cayre M, Metellus P, Beclin C, Ouafik L, Figarella-Branger D (2009) A2B5 cells from human glioblastoma have cancer stem cell properties. Brain Pathol (in press)

18. van den Bent MJ, Vogelbaum MA, Wen PY, Macdonald DR, Chang SM (2009) End point assessment in gliomas: novel treatments limit usefulness of classical Macdonald's Criteria. J Clin Oncol 27(18):2905-2908

19. Varghese M, Olstorn H, Sandberg C, Vik-Mo EO, Noordhuis P, Nistér M, Berg-Johnsen J, Moe MC, Langmoen IA (2008) A comparison between stem cells from the adult human brain and from brain tumors. Neurosurgery 63(6):1022-1033

20. Walker MD, Alexander E Jr, Hunt WE et al (1978) BTSG 6901 Evaluation of BCNU and/or radiotherapy in the treatment of anaplastic gliomas. A cooperative clinical trial. J Neurosurg 49 (3):333-343

21. Wang MY, Lu KV, Zhu S, Dia EQ, Vivanco I, Shackleford GM, Cavenee WK, Mellinghoff IK, Cloughesy TF, Sawyers CL, Mischel PS (2006) Mammalian target of rapamycin inhibition promotes response to epidermal growth factor receptor kinase inhibitors in PTEN-deficient and PTEN-intact glioblastoma cells. Cancer Res 66(16):7864-7869

22. Watanabe T, Nobusawa S, Kleihues P, Ohgaki H (2009) IDH1 mutations are early events in the development of astrocytomas and oligodendrogliomas. Am J Pathol 174(4):1149-1153

23. Yan H, Parsons DW, Jin G, McLendon R, Rasheed BA, Yuan W, Kos I, Batinic-Haberle I, Jones S, Riggins GJ, Friedman H, Friedman A, Reardon D, Herndon J, Kinzler KW, Velculescu VE, Vogelstein B, Bigner DD (2009) IDH1 and IDH2 mutations in gliomas. N Engl J Med 360(8):765-773 